\title{
Adipocyte-Lineage Cells Support Growth and Dissemination of Multiple Myeloma in Bone
}

\author{
Timothy N. Trotter, * Justin T. Gibson, ${ }^{*}$ Tshering Lama Sherpa, ${ }^{*}$ Pramod S. Gowda, ${ }^{*}$ Deniz Peker, ${ }^{*}$ and Yang Yang ${ }^{* \dagger}$
}

From the Department of Pathology* and the Comprehensive Cancer Center and the Center for Metabolic Bone Disease, ${ }^{\dagger}$ University of Alabama at Birmingham, Birmingham, Alabama

Accepted for publication July 19, 2016.

Address correspondence to Yang Yang, M.D., Ph.D., Department of Pathology, University of Alabama at Birmingham, WTI 320A, 1824 Sixth Ave. S., Birmingham, AL 35294. E-mail: yangyang@ uabmc.edu.

\begin{abstract}
Multiple myeloma (MM) cells reside in the bone marrow microenvironment and form complicated interactions with nonneoplastic, resident stromal cells. We previously found that aggressive MM cells shift osteoblast progenitors toward adipogenesis. In addition, adipocytes are among the most common cell types in the adult skeleton; both mature adipocytes and preadipocytes serve as endocrine cells that secrete a number of soluble molecules into the microenvironment. Therefore, we used a combination of in vivo and in vitro methods to test the hypothesis that an increase in adipocyte lineage cells feeds back to promote MM progression. The results of this study revealed that bone marrow from patients with MM indeed contains increased preadipocytes and significantly larger mature adipocytes than normal bone marrow. We also found that preadipocytes and mature adipocytes secrete many molecules important for supporting $M M$ cells in the bone marrow and directly recruit MM cells through both monocyte chemotactic protein-1 and stromal cell-derived factor-1 $\alpha$. Co-culture experiments found that preadipocytes activate Wnt signaling and decrease cleaved caspase-3, whereas mature adipocytes activate ERK signaling in MM cells. Furthermore, mature adipocyte conditioned medium promotes MM growth, whereas co-culture with preadipocytes results in enhanced MM cell chemotaxis in vitro and increased tumor growth in bone in vivo. Combined, these data reveal the importance of preadipocytes and mature adipocytes on MM progression and represent a unique target in the bone marrow microenvironment. (Am J Pathol 2016, 186: 3054-3063; http://dx.doi.org/10.1016/j.ajpath.2016.07.012)
\end{abstract}

Multiple myeloma (MM) is a malignancy of plasma cells that localizes to the bone marrow (BM), where it depends on the microenvironment for survival, growth, drug resistance, and dissemination (metastasis). ${ }^{1-4}$ Although new therapies that counteract the supportive effects of the microenvironment, such as lenalidomide and bortezomib, have improved patient survival in recent years, understanding how MM cells interact with other cells in the BM is crucial for designing more effective treatments. ${ }^{5,6}$ Numerous changes occur in the BM microenvironment as a response to MM cells, both at the primary tumor site and in distant sites. ${ }^{7,8}$ The establishment of a premetastatic niche, in which distant sites become hospitable to cancer cells before metastasis occurs, is emerging as an important step in the progression of many cancers, including MM. ${ }^{9}$ These alterations include enhanced angiogenesis, bone destruction, and modulation of immune cells, although the exact mechanisms driving these changes and the relative effect on MM pathogenesis remain unclear. ${ }^{10}$

It is widely accepted that MM cells inhibit osteoblast differentiation and function, resulting in an uncoupling of bone formation and resorption. ${ }^{11}$ However, we previously reported that $\mathrm{MM}$ cells not only inhibit osteoblastogenesis

\footnotetext{
Supported by NIH grant R01CA151538 (Y.Y.), an International Myeloma Foundation Senior Award (Y.Y.), University of Alabama at Birmingham Center for Metabolic Bone Disease pilot grant (Y.Y.), and a Carmichael Scholarship Award (T.N.T.).

Disclosures: None declared.

An abstract based on a part of this work was presented as a poster at the 57th Annual Meeting of American Society of Hematology, December 6-9, 2015, Orlando, FL.

A guest editor acted as the editor-in-chief for this article. No person at the University of Alabama at Birmingham was involved in the peer review process or final disposition of this article.
} 
but also shift osteoblast progenitors toward adipogenesis. ${ }^{8}$ Importantly, these changes also occur in distant bone sites before arrival of disseminating MM cells through soluble molecules secreted by MM cells. ${ }^{8}$ Because both preadipocytes and mature, lipid-filled adipocytes are highly secretory, releasing a number of cytokines and hormones locally and systemically, we hypothesized that this increased adipogenesis in BM promotes $\mathrm{MM}$ progression and dissemination to new bone sites. ${ }^{12}$ In the present study, we tested this hypothesis using in vivo and in vitro models and determined mechanisms of adipocyte action in MM.

\section{Materials and Methods}

\section{Cell Lines and Reagents}

3T3-L1 mouse preadipocytes were maintained at $70 \%$ to 80\% confluence in Dulbecco's modified Eagle's medium (DMEM) (Corning, Corning, NY) supplemented with $10 \%$ calf serum, $1 \%$ penicillin/streptomycin, and $1 \%$ L-glutamine in a $37^{\circ} \mathrm{C}$ incubation chamber at $5 \% \mathrm{CO}_{2}$. 5TGM1 or 5TGM1-luc mouse MM cells were grown in RPMI 1640 medium (Corning) supplemented with $10 \%$ fetal bovine serum (FBS), $1 \%$ penicillin/streptomycin, and 1\% L-glutamine in a $37^{\circ} \mathrm{C}$ incubation chamber at $5 \% \mathrm{CO}_{2}$.

Mouse phosphorylated ERK, total ERK, unphosphorylated $\beta$-catenin (active $\beta$-catenin), total $\beta$-catenin, and cleaved caspase- 3 antibodies were purchased from Cell Signaling Technology (Danvers, MA; catalog numbers 4370, 4695, 8814, 9562, and 9664, respectively). Mouse $\beta$-actin antibody was purchased from Sigma-Aldrich (St. Louis, MO; catalog number A5316). Mouse monocyte chemotactic protein (MCP)-1, stromal cell-derived factor (SDF)- $1 \alpha$, goat IgG isotype control, mouse IgG1 isotype control antibodies, and recombinant human preadipocyte factor (Pref)-1 were purchased from R\&D Systems (Minneapolis, MN; catalog numbers AF-479-NA, MAB310, AB-108-C, MAB002, and 1144-PR-025, respectively). Human Pref-1 antibody and mouse osteopontin (OPN), MCP-1, and SDF- $1 \alpha$ enzymelinked immunosorbent assay (ELISA) kits were purchased from Abcam (Cambridge, MA; catalog numbers ab21682, ab100734, ab100721, and ab100741, respectively). Mouse

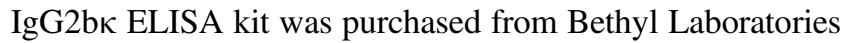
Inc. (Montgomery, TX; catalog number E90-109).

\section{Adipocyte Differentiation and Adipocyte/MM Cell Co-Culture}

3T3-L1 preadipocytes were seeded in 6-well plates at $4 \times 10^{4}$ cells $/ \mathrm{cm}^{2}$ in DMEM that contained $10 \%$ calf serum. When cells reached confluence, medium was replaced and cells were cultured for an additional 2 days. Cells were induced toward mature adipocytes with a differentiation medium that contained DMEM with $10 \%$ FBS, $1.5 \mu \mathrm{g} / \mathrm{mL}$ of insulin, $1 \mu \mathrm{mol} / \mathrm{L}$ of dexamethasone, and $0.5 \mathrm{mmol} / \mathrm{L}$ 3-isobutyl-1-methylxanthine (day 0). Medium was replaced with DMEM that contained $10 \%$ FBS and $1.5 \mu \mathrm{g} / \mathrm{mL}$ of insulin (day 2), followed by DMEM that contained $10 \%$ FBS only for the remainder of differentiation (day 4). All experiments with mature adipocytes began at day 10 on which adipocytes were considered mature. These cells contained large lipid vacuoles and accounted for $\geq 95 \%$ of the cell population.

For cell co-culture, inserts containing $0.45-\mu \mathrm{m}$ pores (BD Biosciences, Bedford, MD) were placed in 6-well plates that contained subconfluent 3T3-L1 preadipocytes, mature adipocytes, or media only in the bottom well. Then $6 \times 10^{5}$ 5TGM1 MM cells were added to each insert and incubated at $37^{\circ} \mathrm{C}$ and $5 \% \mathrm{CO}_{2}$ for 3 days. Co-cultured $\mathrm{MM}$ cells were then collected for use in experiments. This co-culture system did not allow cell-cell contact; adipocytes and MM cells communicated through soluble factors only.

\section{Western Blot Analysis}

Equal amounts of protein $(100 \mu \mathrm{g})$ were subjected to $4 \%$ to $12 \%$ gradient SDS-PAGE (BioRad, Hercules, CA) and transferred to a nitrocellulose membrane (Schleicher and Schuell, Dassel, Germany). Transferred proteins were probed with appropriate antibodies and visualized using an enhanced chemiluminescence system (GE Health Care, Little Chalfont, UK). Densitometric analysis was performed using ImageJ version 1.49u (NIH, Bethesda, MD; http:// imagej.nih.gov/ij).

\section{TGM1 Cell I.V. Injection Animal Model}

C57BL/KaLwRij mice for i.v. injection of syngeneic 5TGM1-luc mouse MM cells were purchased from Harlan Laboratories, Inc. Many aspects of human MM are faithfully replicated in this mouse model of MM..$^{13-15}$ 5TGM1 cells constitutively expressing luciferase and co-cultured as described were injected into 6-week-old, male, C57BL/ KaLwRij mice via lateral tail veins. Serum was collected biweekly, and the levels of $\operatorname{IgG} 2 \mathrm{~b} \kappa$ (a soluble marker of 5TGM1 cells) were measured by ELISA. Weekly bioluminescent imaging also noninvasively tracked the extent of bone-homing and tumor growth in vivo. ${ }^{16}$ All animal studies were performed in accordance with $\mathrm{NIH}$ guidelines and approved by the University of Alabama at Birmingham (UAB) Institutional Animal Care and Use Committee.

\section{Generation of CM}

3T3-L1 preadipocytes were seeded in 6-well plates and cultured until approximately $70 \%$ confluent. Old medium was aspirated, cells were washed twice with phosphatebuffered saline (PBS), and DMEM supplemented with 10\% FBS was added. Conditioned medium (CM) was collected after 48 hours, centrifuged to remove cellular debris, filtered through a $0.22-\mu \mathrm{m}$ syringe filter, and stored at $-80^{\circ} \mathrm{C}$ until further use. For mature adipocyte $\mathrm{CM}$, on day 10 of 
differentiation, cells were washed twice with PBS, and media was replaced with DMEM supplemented with $10 \%$ FBS. CM was collected after 48 hours, centrifuged, filtered, and stored at $-80^{\circ} \mathrm{C}$ until further use.

\section{Migration Assays}

5TGM1 cells co-cultured with preadiopcytes or mature adipocytes as described were collected and washed once with PBS, and $2 \times 10^{5}$ cells in $0.5 \mathrm{~mL}$ of serum-free medium were added to $8-\mu \mathrm{m}$ pore size Transwell inserts (Corning) in triplicate. Cells were allowed to migrate toward complete medium in the bottom chamber at $37^{\circ} \mathrm{C}$ and $5 \% \mathrm{CO}_{2}$. Migrated cells in the bottom chambers were enumerated after 24 hours using a Z1 Dual Threshold Coulter Counter (Beckman Coulter, Brea, CA). ${ }^{17}$

In a separate set of experiments, 5TGM1 cells cultured alone were collected and washed twice with PBS, and $2 \times 10^{5}$ cells in $0.5 \mathrm{~mL}$ serum-free medium were added to $8-\mu \mathrm{m}$ pore inserts. Cells were allowed to migrate toward fresh DMEM with $10 \%$ FBS, preadipocyte CM, or mature adipocyte $\mathrm{CM}$ in the bottom chambers in triplicate. Migrated cells were enumerated after 24 hours using a Z1 Dual Threshold Coulter Counter. For cytokine inhibition studies, anti-SDF-1 $\alpha$, anti-MCP-1 neutralizing antibodies, or appropriate isotype controls were added to fresh medium or adipocyte $\mathrm{CM}$ and incubated at $37^{\circ} \mathrm{C}$ for 30 minutes before use. Recombinant human Pref-1 was added directly to fresh medium before migration assays.

\section{ELISA}

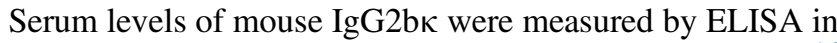
duplicate according to the manufacturer's instructions. ${ }^{16}$ OPN, MCP-1, and SDF- $1 \alpha$ levels in CM of preadipocytes and mature adipocytes were measured using mouse OPN, MCP-1, and SDF-1 $\alpha$ ELISA kits. All assays were performed according to the manufacturer's protocols with each sample measured in duplicate.

\section{Cytokine and Chemokine Array}

A customized cytokine and chemokine array (RayBiotech, Norcross, GA), which uses a chemiluminescent sandwich ELISA format and probes for 29 cytokines, chemokines, and growth factors commonly related to bone-homing and growth of tumor cells, was used according to the manufacturer's recommendations. Film scans were analyzed by densitometry using Gilles Carpentier's protein array analyzer macro for ImageJ.

\section{Immunohistochemistry and Adipocyte Quantification}

All biopsy procedures and immunohistochemistry protocols were approved by the UAB Institutional Review Board. Paraffin-embedded BM core biopsy specimens from 14 age- and weight-matched normal BM donors and 35 untreated patients with MM, obtained from the Department of Pathology at UAB, were stained with hematoxylin and eosin (H\&E) or with anti-human Pref-1 antibodies. ${ }^{18}$ Balloon-like mature adipocytes (H\&E staining) and preadipocytes, which were Pref-1 positive and had fibroblastic morphologic characteristics, were enumerated using ImageJ in five representative views per slide. Adipocyte area in $\mathrm{H} \& \mathrm{E}$ stained slides was calculated using ImageJ and the method described previously. ${ }^{19}$

\section{Statistical Analysis}

Statistical comparisons between two experimental groups were analyzed by $t$-test. For comparisons among multiple groups, analysis of variance followed by a post hoc Bonferroni correction was used. Data were considered significantly different when $P<0.05$ and are reported as such.

\section{Results}

\section{Patients with MM Have Enhanced Bone Marrow Adipogenesis}

To determine the clinical relevance of our previous findings, we evaluated the BM of 14 healthy BM donors and 35 untreated patients with MM. Paraffin-embedded BM biopsy specimens were stained with $\mathrm{H} \& \mathrm{E}$ and for Pref-1, a welldescribed marker for preadipocytes. ${ }^{18,20}$ Balloon-like mature adipocytes and Pref-1-positive preadipocytes were enumerated. The results revealed significantly greater Pref1-positive cells in the BM of patients with MM compared with healthy BM, indicating an increase in the preadipocyte population in MM patients (Figure 1, A and B). Importantly, we did not observe Pref-1 staining of malignant plasma cells in the BM. The absolute number of mature adipocytes was higher in MM patients, but the finding did not reach statistical significance. However, a significant increase in adipocyte size was observed in the BM of patients with $\mathrm{MM}$ compared with healthy BM (Figure 1, C and D). In addition, large adipocytes represented a greater percentage of the total adipocyte area in MM patients compared with healthy BM (Figure 1E). Together, these data indicate that the adipocytic population is altered in the $\mathrm{BM}$ of MM patients.

\section{Adipocyte Lineage Cell-Derived Soluble Molecules Directly Attract MM Cells}

We have previously found that $\mathrm{MM}$ cells can redirect osteoblast progenitors toward adipogenesis in distant bone sites. ${ }^{8}$ Adipocytes and precursor cells secrete soluble molecules into the local microenvironment and bloodstream that serve to attract other cells, such as macrophages. ${ }^{21}$ Therefore, we first determined whether preadipocytes and/or mature adipocyte-derived soluble molecules can directly attract MM cells. Transwell migration assays were 
A

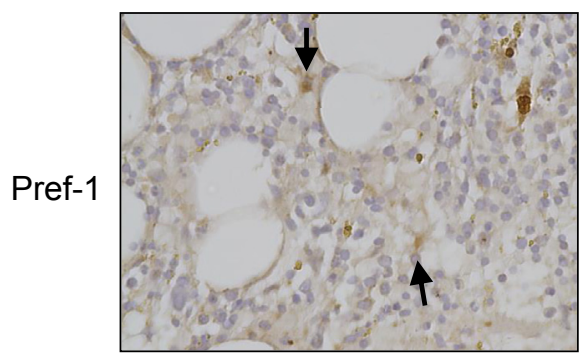

C

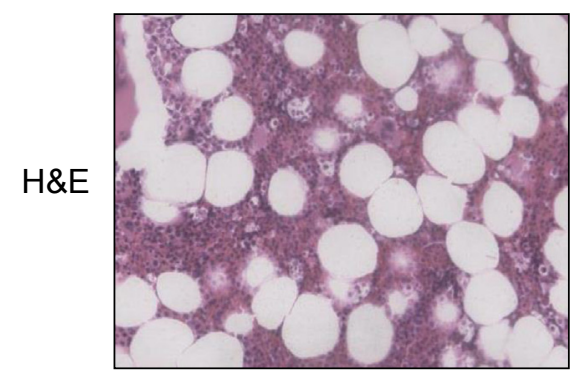

E

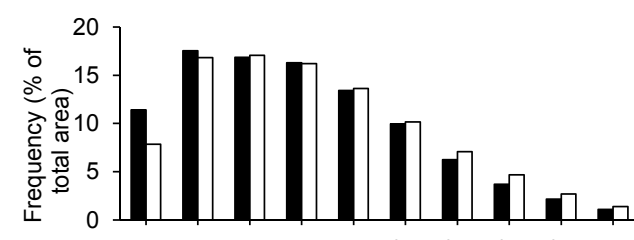

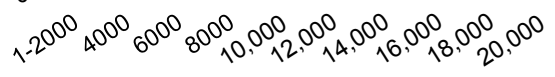

MM
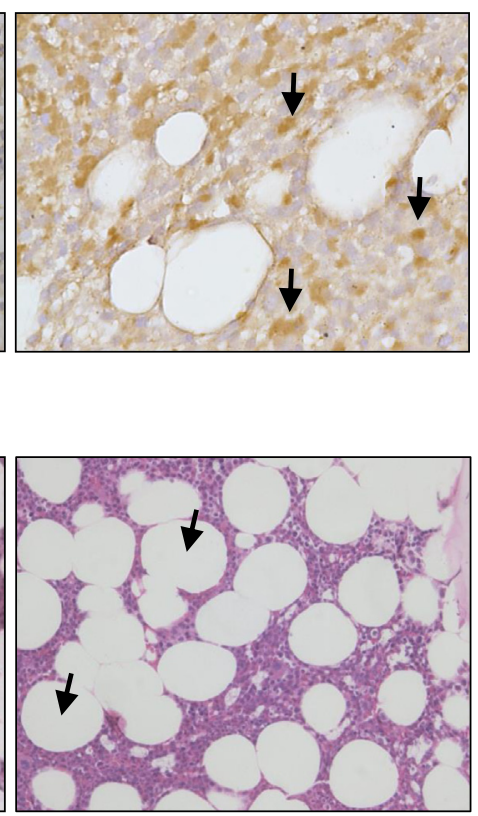

B
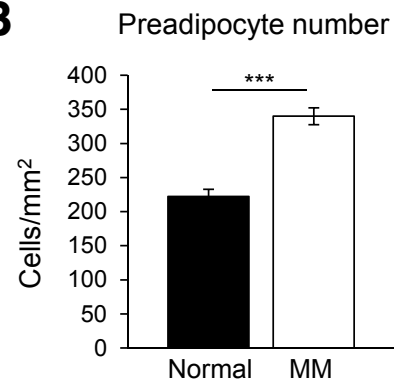

D

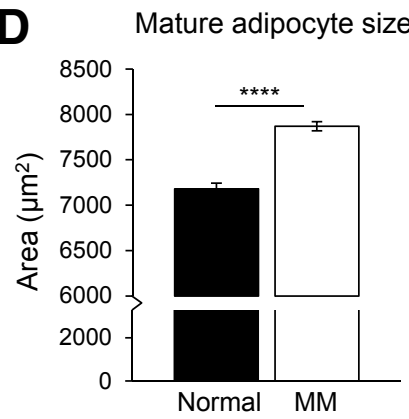

Normal $\square \mathrm{MM}$

Figure 1 Adipocyte lineage cells are altered in the bone marrow (BM) of patients with multiple myeloma (MM) compared with normal BM. A: BM biopsy specimens from healthy individuals and MM patients were stained for the preadipocyte marker preadipocyte factor (Pref-1). Cells positive for Pref-1 (arrows) were more abundant in MM patients compared with healthy BM specimens. Representative images are shown. B: Enumeration of Pref-1-positive cells from five random fields of view per section. C: Hematoxylin and eosin (H\&E)-stained BM biopsies from healthy BM donors and MM patients. Very large adipocytes were more frequently observed in MM patients (arrows). D: Mature adipocyte area quantified from five random fields of view per section. Adipocytes in MM bone marrow were significantly larger than normal bone marrow. E: Distribution of mature adipocyte size as percentage of total adipocyte area. A higher percentage of larger

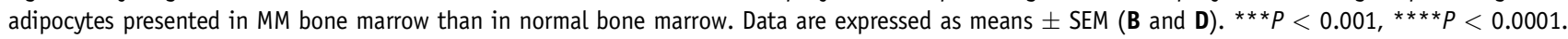

performed and revealed that both preadipocyte and mature adipocyte CM stimulated chemotaxis of mouse 5TGM1 (Figure 2A) and human CAG (Figure 2B) MM cells significantly more than fresh medium alone. Interestingly, MM cells consistently had more chemoattraction toward preadipocyte $\mathrm{CM}$ than to mature adipocyte $\mathrm{CM}$; however, this finding did not always reach statistical significance. These data confirm that preadipocytes and mature adipocytes directly attract MM cells through soluble molecules.

\section{Identification of Soluble Molecules Secreted by Adipocyte Lineage Cells Responsible for Stimulating MM Cell Migration}

To determine soluble molecules responsible for chemoattraction of MM cells to preadipocyte and mature adipocyte CM, we performed a cytokine array, consisting of 29 cytokines, chemokines, or growth factors important for MM bone dissemination and growth, on preadipocyte and mature adipocyte CM. We found that both preadipocytes and mature adipocytes secrete a number of pro-MM molecules (Figure 3A). Interestingly however, CM from preadipocytes contained higher concentrations of many of these factors. Notably, hepatocyte growth factor, MCP-1, OPN, vascular endothelial growth factor, and SDF-1 $\alpha$, all supporters of MM progression, were secreted at relatively high levels (Figure 3, $\mathrm{A}$ and B). ${ }^{22-25}$ Selected molecules (SDF-1 $\alpha$, MCP-1, and OPN) were tested quantitatively by ELISA, confirming the existence of these molecules in the CM of both preadipocytes and mature adipocytes and increased abundance in preadipocyte CM compared with mature adipocyte CM (Figure 3C). These data reveal that both preadipocytes and mature adipocytes secrete molecules that are known to support MM progression through multiple pathways.

We also wanted to determine preadipocyte- and mature adipocyte-derived molecules contributing to attraction of 


\section{A}

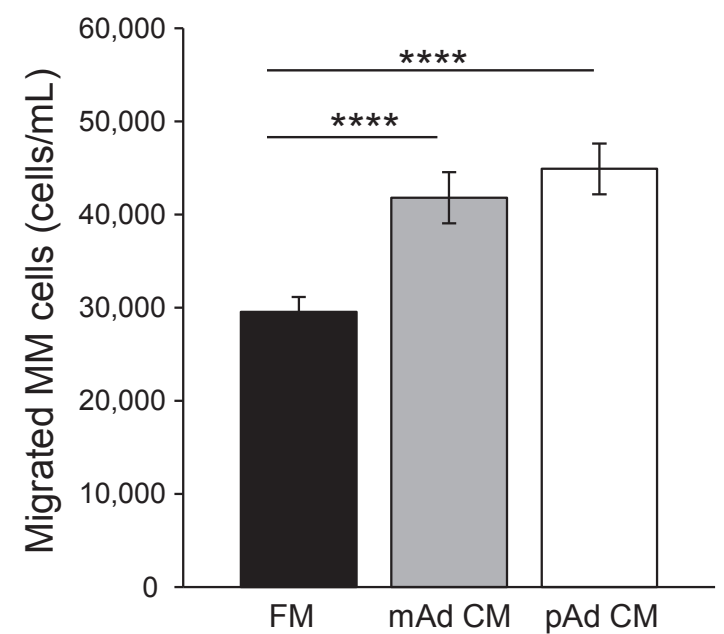

B

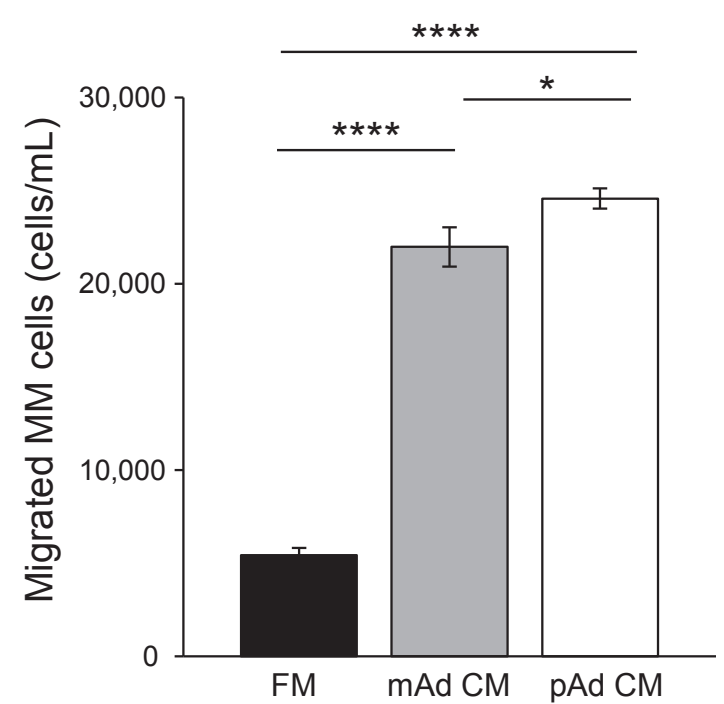

Figure 2 Adipocyte lineage cells secrete soluble molecules that attract multiple myeloma (MM) cells. A: Numbers of migrated mouse 5TGM1 MM cells. Preadipocyte (pAd) conditioned medium (CM), mature adipocyte (mAd) CM, or fresh medium (FM) were added in the bottom of each well as chemoattractants and 5TGM1 cells seeded in the inserts. The number of 5TGM1 cells migrated into the bottom of each well was counted after 24 hours. MM cells migrated significantly more toward both preadipocyte and mature adipocyte CM than to fresh medium, and preadipocyte CM consistently had the greatest chemoattraction. B: Similar results were observed using human CAG MM cells. Each sample was analyzed in triplicate and each assay was performed 3 times. Data are expressed as means \pm SEM. ${ }^{*} P<0.05,{ }^{* * * *} P<0.0001$.

MM cells. MCP-1 and particularly SDF- $1 \alpha$ are important chemoattractants for $\mathrm{MM}$ cell homing to the $\mathrm{BM}^{25,26}$ Because we found that both molecules are secreted by preadipocytes and mature adipocytes, we repeated MM cell migration assays (Figure 2) with preadipocyte or mature adipocyte CM pretreated with MCP-1 or SDF-1 $\alpha$ neutralizing antibodies or the respective isotype control. MM cell chemotaxis was significantly decreased toward both preadipocyte and mature adipocyte $\mathrm{CM}$ that contained MCP-1 $(12 \mu \mathrm{g} / \mathrm{mL})$ (Figure 3D) or SDF-1 $\alpha(25 \mu \mathrm{g} / \mathrm{mL})$ neutralizing antibodies (Figure 3E). Because Pref-1 can be cleaved from the cell surface and released into the microenvironment and because Pref- 1 is expressed by preadipocytes, we performed migration assays using recombinant Pref- 1 as the chemoattractant. ${ }^{27}$ Interestingly, a dose-dependent increase in chemotaxis of 5TGM1 MM cells was seen toward Pref-1 (Figure 3F). Combined, these results indicate that adipocyte lineage cells independently attract MM cells through MCP-1, SDF-1 $\alpha$, and likely Pref-1.

Preadipocytes and Mature Adipocytes Promote MM Cell Aggressiveness via Different Signaling Pathways and Mechanisms

The effect of cross talk between MM cells and preadipocytes or mature adipocytes was investigated using a Boyden chamber co-culture system. MM cells, separated by a membrane that contained $0.45-\mu \mathrm{m}$ pores, were cultured with medium only, preadipocytes, or mature adipocytes in the bottom chamber for 3 days. We initially wanted to determine whether adipocyte lineage cells activate specific signaling pathways in MM cells; therefore, Western blot analysis for signaling pathway activity was performed on MM cells after 3 days of co-culture. Interestingly, preadipocytes and mature adipocytes activated different pathways in MM cells. Of the pathways tested, MM cells educated by preadipocytes consistently revealed active (unphosphorylated) $\beta$-catenin (Wnt signaling) and decreased cleaved caspase-3 levels, whereas MM cells educated by mature adipocytes had an activation of ERK signaling (Figure 4, A and B). Both Wnt and ERK signaling are reported to support MM progression, and decreased cleaved caspase-3 suggests an inhibition of apoptosis. MM cell migration assays after co-culture were also performed to determine any functional changes in MM cells. ${ }^{10,16}$ After 24 hours, MM cells previously cultured with preadipocytes migrated significantly more than those cultured alone or with mature adipocytes. However, coculture with mature adipocytes did not alter the motility of MM cells (Figure 4C). On the other hand, cell counting revealed that $\mathrm{MM}$ cells cultured in the presence of mature adipocyte $\mathrm{CM}$ grew at an increased rate compared with those grown in preadipocyte $\mathrm{CM}$ or fresh medium (Figure 4D). Together, these data suggest tht pre-adipocytes and mature adipocytes both support MM progression through independent but complementary mechanisms.

\section{Preadipocytes Promote MM Dissemination and Growth in Vivo}

Because MM cells had alterations in behavior and signaling after co-culture with preadipocytes or mature adipocytes, we decided to test whether this had sustained effects in vivo. 5TGM1-luc MM cells were cultured with preadipocytes, 
A

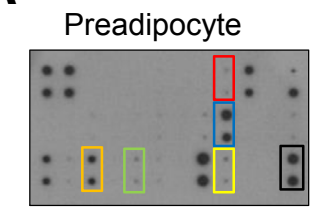

$\square$ HGF $\square$ MCP-1 $\square$ VEGF $\square$ OPN $\square$ SDF-1 $\square$ Positive control
B

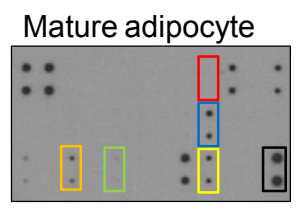

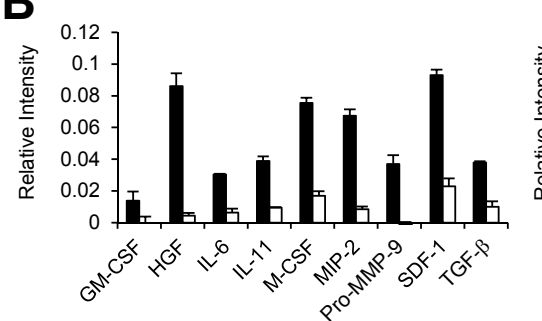

SDF-1a
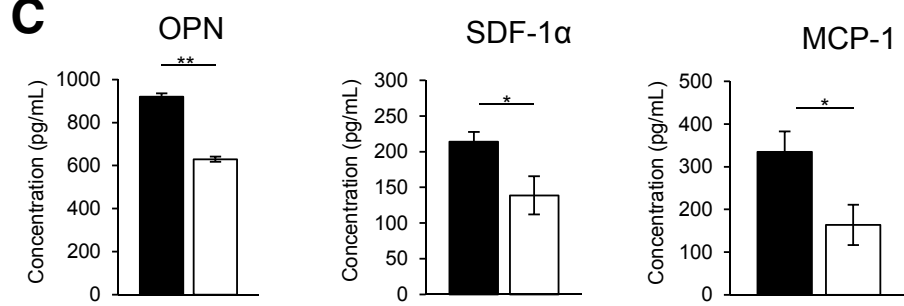

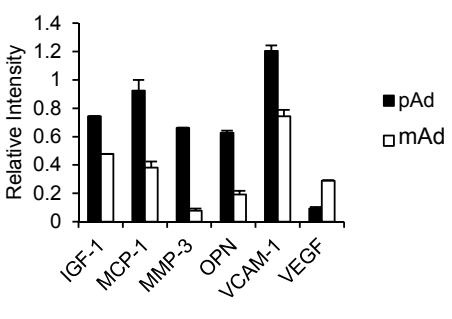

pAd

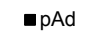

amAd
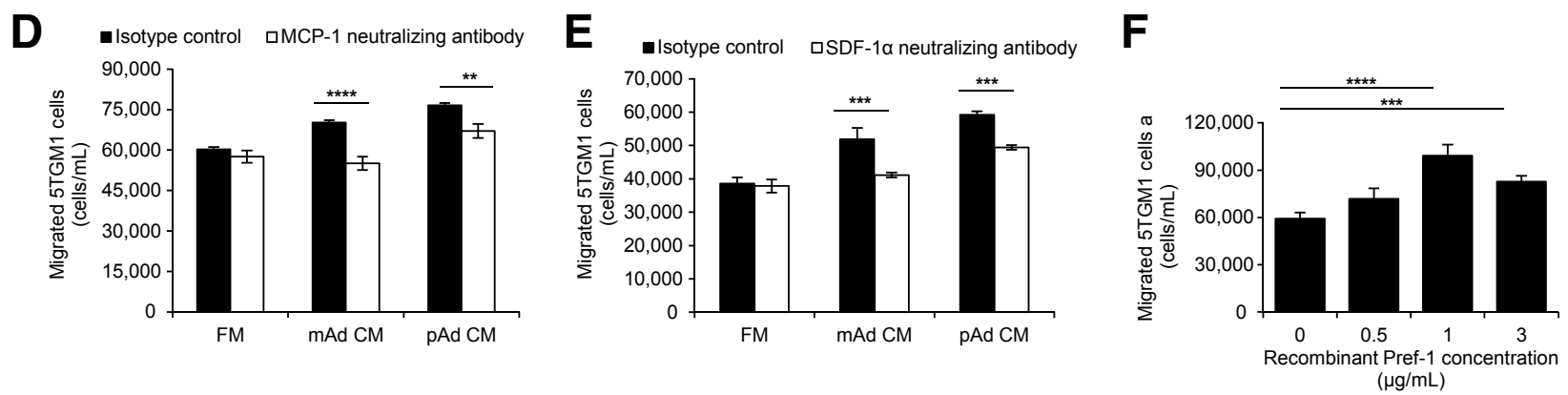

Figure 3 Adipocytes secrete soluble molecules known to promote multiple myeloma (MM) progression and directly attract MM cells through monocyte chemotactic protein (MCP)-1 and stromal cell-derived factor (SDF)-1 $\alpha$. A: Cytokine array of conditioned medium (CM) collected from 3T3-L1 preadipocytes (pAds) or mature adipocytes (mAds). Molecules of particular importance are highlighted. B: Quantification of pAds versus mAds. C: Enzyme-linked immunosorbent assays for osteopontin (OPN), MCP-1, and SDF- $1 \alpha$ in pAd and mAd CM confirmed cytokine array results. Each assay was analyzed in duplicate. D: Neutralizing antibodies against MCP-1 or appropriate isotype control were added to CM from preadipocytes, mature adipocytes, or fresh media (FM). Neutralizing MCP-1 resulted in significantly reduced migration of mouse 5TGM1 MM cells toward both pAd and mAd CM compared with isotype control. E: Similar results were observed when adding a SDF-1 $\alpha$ neutralizing antibodies. F: Recombinant human preadipocyte factor (Pref-1) dose dependently acted as a chemoattractant for MM cells. Each sample was analyzed in triplicate, and each assay was performed at least 2 times. Data are expressed as means \pm SEM. ${ }^{*} P<0.05,{ }^{*} P<0.01,{ }^{* *} P<0.001$, and ${ }^{* * * * P}<0.0001$. GM-CSF, granulocyte-macrophage colony-stimulating factor; HGF, hepatocyte growth factor; IGF1, insulin-like growth factor-1; M-CSF, macrophage colony-stimulating factor; MIP-2, macrophage inflammatory protein-2; MMP, matrix metalloproteinase; TGF- $\beta$, transforming growth factor- $\beta$; VCAM-1, vascular cell adhesion molecule- 1 ; VEGF, vascular endothelial growth factor.

mature adipocytes, or fresh medium alone for 3 days as described. C57B1/KaLwRij mice ( $n=7$ per group) were then injected with $1.5 \times 10^{6} \mathrm{MM}$ cells in $150 \mu \mathrm{L}$ of PBS via tail vein. Tumor burden was tracked by bioluminescent

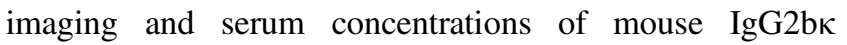
(secreted by 5TGM1 cells and correlates well with wholebody tumor burden). ${ }^{13} \mathrm{MM}$ cells cultured with preadipocytes could be detected in bone by bioluminescent imaging as early as 2 weeks, whereas mice injected with MM cells cultured alone had no detectable tumors at this point. This trend continued at 4 weeks (Figure 5A). By 6 weeks mice in both groups had detectable tumors via bioluminescent imaging, but tumors in the preadipocyte co-culture group were significantly larger (Figure 5A).

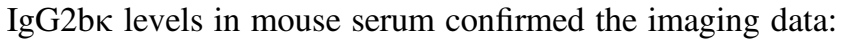
mice injected with MM cells co-cultured with preadipocytes had significantly higher tumor burden than those injected with MM cells cultured alone (Figure 5B). Interestingly, no difference was observed in tumor burden in mice injected with MM cells precultured alone or precultured with mature adipocytes, indicated by $\mathrm{IgG} 2 \mathrm{~b} \kappa$ concentrations in mouse serum (Figure 5C). These data indicate that preadipocytes induce lasting changes in MM cells that support metastasis and growth.

\section{Discussion}

MM cells in the BM form complex relationships with neighboring and distant cells, resulting in an environment that allows MM to thrive. ${ }^{10}$ Osteoblasts and adipocytes are two dominant cell types in the BM and are both derived from mesenchymal stem cells. ${ }^{28}$ In bone, these two pathways appear to be inversely related, illustrated by the loss of bone and increase of fat in BM during aging or osteoporosis. ${ }^{29,30}$ Many studies have found that osteoblasts are 


\section{A}

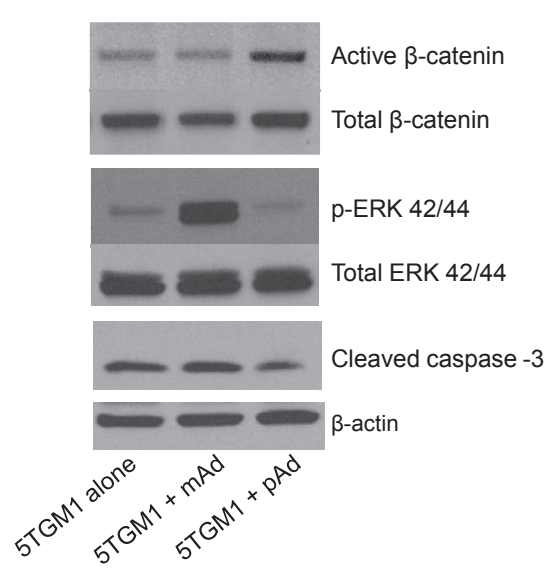

C

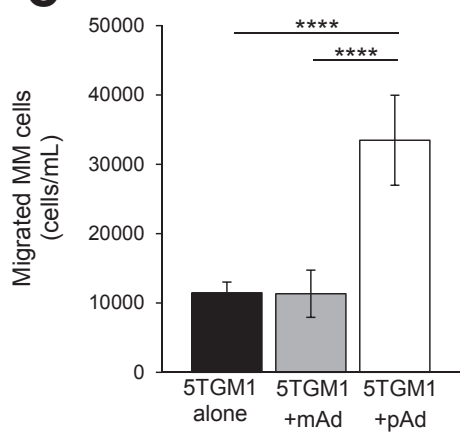

B
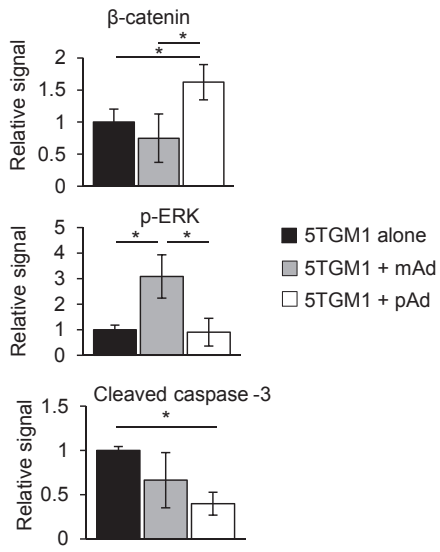

D

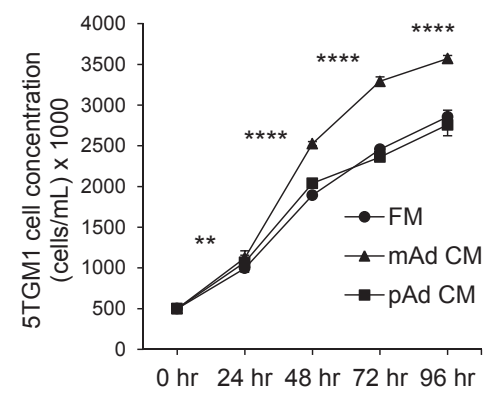

Figure 4 Adipocyte lineage cells differentially alter behavior and signaling of multiple myeloma (MM) cells. A: Western blotting after MM cell co-culture with preadipocytes (pAds) or mature adipocytes (mAds) for 3 days. The pAds activated $\beta$-catenin signaling and decreased cleaved caspase-3 levels in 5TGM1 MM cells, whereas the mAds activated ERK signaling in MM cells compared with those cultured alone. B: Quantification of Western blots from three independent experiments. C: MM cell migration assay. 5TGM1 MM cells cultured with pAds revealed enhanced migration compared with those cultured with mAds or in fresh medium (FM) alone. D: Cell proliferation rates. 5TGM1 MM cells cultured in mAd conditioned medium (CM) revealed increased growth compared with those cultured with pAd CM or FM. Each sample was analyzed in triplicate, and each assay was performed three times. Data are expressed as means \pm SEM. ${ }^{*} P<0.05$ ${ }^{* *} P<0.01$, and ${ }^{* * * *} P<0.0001$. p-ERK, phosphorylated ERK. inhibited in $\mathrm{MM}$, and our group has further found that MM cells induce an osteoblastogenesis to adipogenesis switch in osteoblast progenitor cells locally and systemically via secreting soluble factors, such as Dickkopf-related protein 1 (DKK1). ${ }^{8,11,31}$

We initially examined biopsy specimens from MM patients and weight- and age-matched healthy individuals to identify changes in adipocyte lineage cells. Interestingly, we found a significant increase in the number of preadipocytes, which express the preadipocyte marker Pref- 1 , in the BM of MM patients compared with healthy BM. By examining the mature adipocytes, we found a trend toward increased numbers of mature adipocytes, but these cells were significantly larger in MM BM compared with healthy BM. Adipocyte size is positively correlated with inflammatory molecule secretion and lipolysis in adipocytes; therefore, larger adipocytes in MM patient BM may provide both an inflammatory environment and lipids as a fuel source for growing tumor cells. ${ }^{32,33}$ The lack of statistical significance when comparing mature adipocyte number is likely attributable to the limited number of samples evaluated, and as more samples are available we will continue to assess this parameter. These findings support the notion that adipogenesis is enhanced in the BM of MM patients.

Adipocytes, including preadipocytes and mature adipocytes, reportedly secrete cytokines and chemokines that attract inflammatory cells into local tissues, including the BM. ${ }^{34,35}$ We hypothesized that enhanced adipocyte lineage cells in new bone sites would contribute to MM dissemination through similar mechanisms. Indeed, we found that both preadipocyte and mature adipocyte secreted factors could attract MM cells, particularly through MCP-1 and SDF- $1 \alpha$. In addition, we found that both mature adipocytes and preadipocytes secrete many other molecules known for MM growth and bone homing, such as hepatocyte growth factor, IL-6, and vascular endothelial growth factor. ${ }^{22-25}$ Interestingly, however, the preadipocytes secreted much higher amounts of almost all molecules tested, excluding vascular endothelial growth factor. It is known that chemokines secreted by BM cells enable MM cell homing to the bone, and adipocytes are a major constituent in the adult skeleton. ${ }^{30,36}$ The number of adipocyte lineage cells in the bone may therefore strongly contribute to the chemokine gradient for MM homing. Moreover, we found that recombinant Pref- 1 could attract MM cells in a dose-dependent manner. Pref-1 is a membrane protein specifically expressed on preadipocytes that can be cleaved to a soluble form. ${ }^{27}$ This may represent a novel mechanism by which preadipocytes attract MM cells to bone. Studies to further characterize the role of soluble Pref-1 in MM are ongoing.

Because we previously found that MM cells induce adipogenesis through secreted factors, we used a coculture system in which cells could only communicate with soluble molecules to investigate the soluble effects of adipocyte lineage cells on MM cells. ${ }^{8}$ We found that after being cultured with preadipocytes, MM cells migrate more than 


\section{A}

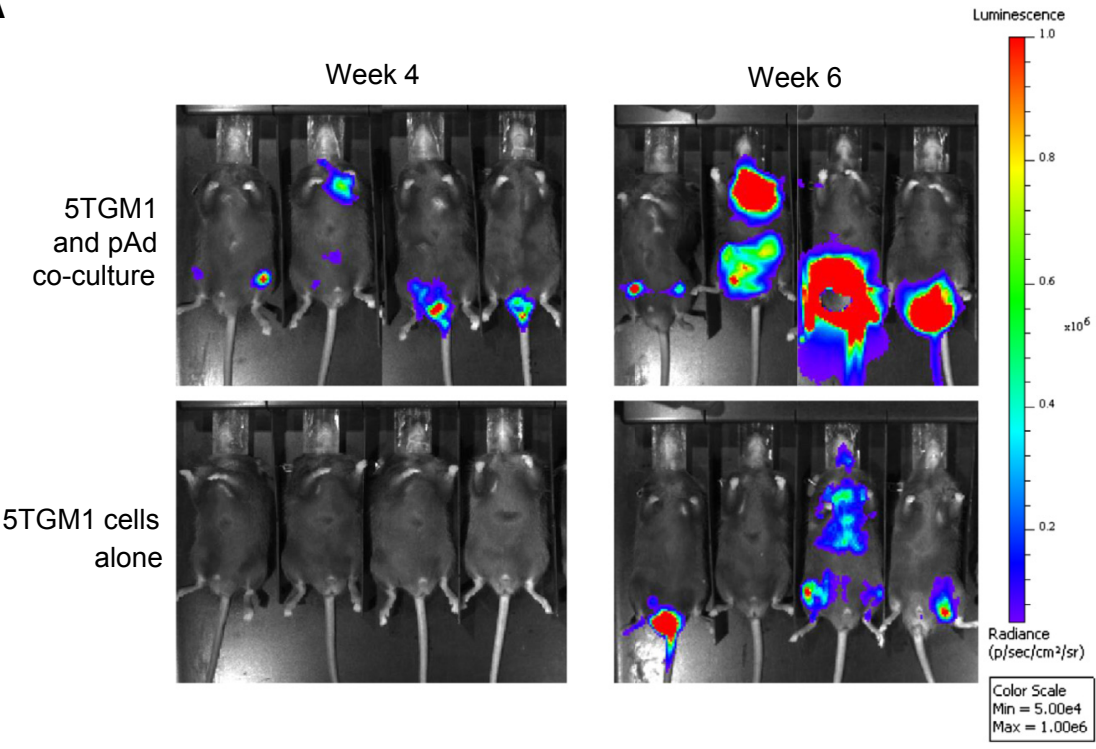

B

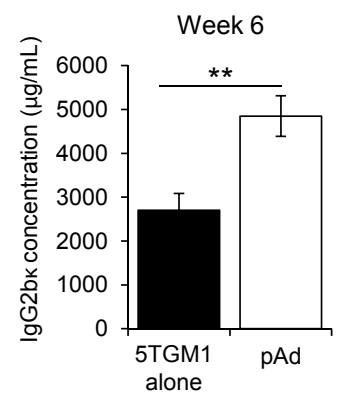

C

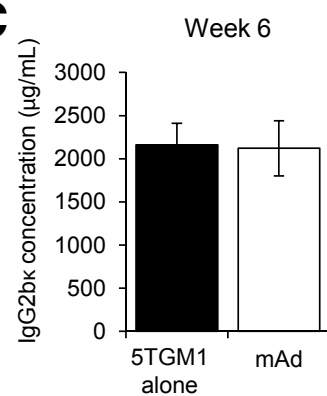

Figure 5 Preadipocyte ( $p A d)$ co-culture promotes myeloma progression in vivo. A: Representative bioluminescent images of mice at 4 weeks (left column) and 6 weeks (right column) after i.v. injection of mouse 5TGM1-luc multiple myeloma (MM) cells cultured alone or with pAds. Tumors were visible earlier and grew larger from pAd co-cultured MM cells compared with MM cells cultured alone. B: IgG2bא serum levels, an indicator of total tumor burden, at time of sacrifice ( 6 weeks) of mice from panel A. C: IgG2bк serum levels in mice injected with mouse 5TGM1-luc MM cells cultured alone or with mature adipocytes (mAds). $n=7$ per group. Data are expressed as means \pm SEM. ${ }^{* *} P<0.01$. those cultured with mature adipocytes or cultured alone. On the other hand, MM cells cultured in mature adipocyte CM proliferated more compared with preadipocyte CM or fresh medium, possibly attributable to adipocyte-derived fatty acids or lactate in the medium, likely energy sources for cancer cells, including $\mathrm{MM} .^{37-39}$ In vivo experiments revealed that prior culture with preadipocytes results in detectable tumors in bone earlier and greater tumor burden overall compared with control. Because MM cells have a decrease in cleaved caspase- 3 and an increase in migration

\section{Primary Bone Site}

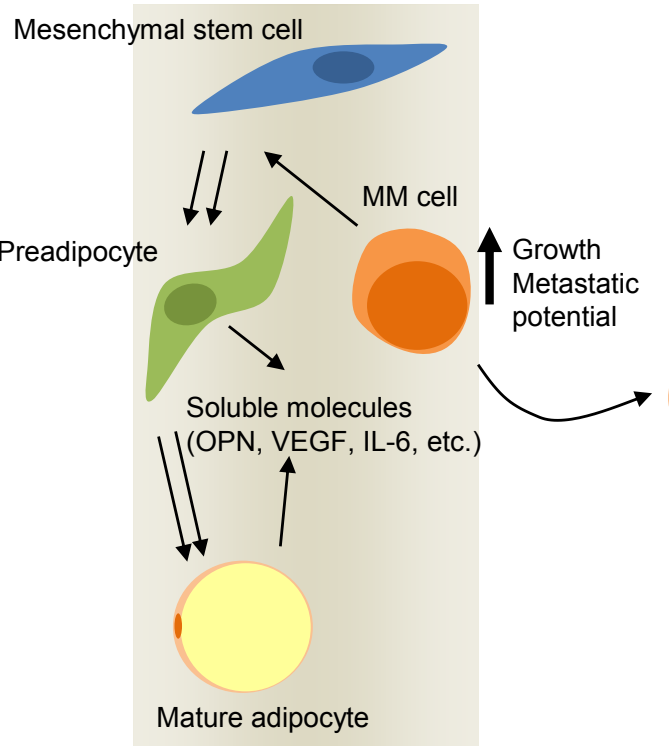

\section{Metastatic Bone Site}

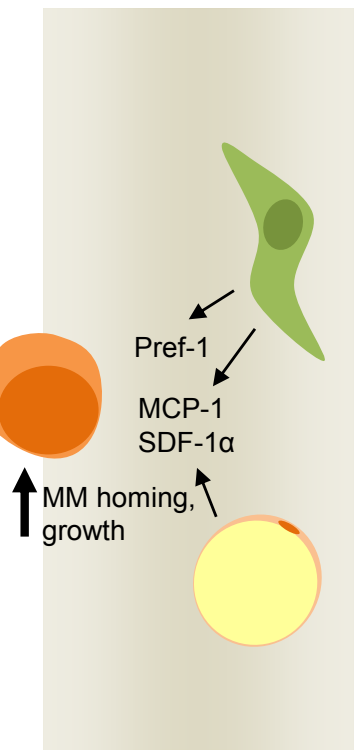

Figure 6 Preadipocytes and mature adipocytes promote multiple myeloma (MM) progression. At the primary bone site, preadipocytes and mature adipocytes likely secrete pro-MM soluble molecules. Constant presence of mature adipocyte-derived molecules enhances MM cell growth, whereas preadipocytes educate MM cells for increased metastasis in new bone sites. In a secondary bone site, enhanced preadipocytes and mature adipocytes secrete chemoattractants, such as monocyte chemotactic protein (MCP)-1, stromal cell-derived factor (SDF)- $1 \alpha$, and preadipocyte factor (Pref)-1, to promote MM homing and subsequent growth. OPN, osteopontin; VEGF, vascular endothelial growth factor. 
ability in vitro after culture with preadipocytes, it is possible that early tumor detection and enhanced tumor burden in vivo is a combined result of enhanced MM infiltration into the bone and survival in these sites. Surprisingly, coculture with mature adipocytes did not enhance the ability of MM cell bone homing and growth in vivo, although mature adipocyte $\mathrm{CM}$ promoted $\mathrm{MM}$ cell migration and proliferation in vitro. These data indicate that preadipocytes and mature adipocytes influence MM cells through different mechanisms and that MM cells may need continual education from mature adipocytes to become more aggressive.

Indeed, the cross talk between MM cells and preadipocytes or mature adipocytes activates different signaling pathways in MM cells. Western blot analyses revealed that $\beta$-catenin signaling is consistently activated in MM cells when cultured with preadipocytes, but ERK signaling is activated in MM cells co-cultured with mature adipocytes. Interestingly, in our hands, this appeared to be mutually exclusive. Wnt/ $\beta$-catenin signaling promotes cancer immune evasion, migration, and survival, possibly mediated by up-regulation of downstream survivin as we have shown previously. ${ }^{16,40-42}$ The ERK pathway is activated by multiple molecules in the BM microenvironment and promotes MM cell proliferation. ${ }^{43,44}$ Preadipocytes also decreased levels of cleaved caspase- 3 in MM cells, suggesting that they activate an anti-apoptotic/survival response in MM cells. ${ }^{45}$ At this point, we are unsure of the specific soluble mediators that define these different signaling profiles; however, future experiments are aimed at this question.

In conclusion, our data reveal that both preadipocytes and mature adipocytes have independent but complementary roles in supporting MM progression and dissemination. On the basis of our data, preadipocytes, and particularly mature adipocytes, could enable MM growth in primary bone sites, whereas preadipocytes induce lasting effects that enhance metastatic potential of MM cells through various soluble molecules (Figure 6). At the same time, these cells in distant bone sites likely secrete chemokines that attract MM cells and support MM cell growth on arrival. Considering the anti-MM effects of osteoblasts on MM cells, it is interesting to speculate that as the BM becomes increasingly adipogenic, whether during the disease or because of other conditions, such as obesity, it also becomes more favorable to MM cells. ${ }^{46,47}$ We previously found that MM cells shift osteoprogenitors toward adipogenesis by secreting DKK1, resulting in decreased Runt-related transcription factor 2 and increased peroxisome proliferator-activated receptor gamma. ${ }^{8,48}$ Therefore, inhibiting DKK1 may provide benefit in reversing these effects and reducing adipocytic support of MM cells. The BM is also home to a variety of other stromal cell populations, and BM mesenchymal stromal cells have been found to contribute to MM progression. Our data indicate that the adipocyte lineage is another important component of this MM-supporting milieu. Future studies will be aimed at uncovering the mediators of preadipocyte-induced metastasis, contact-mediated cross talk of adipocytes and MM cells, and the fundamental differences in the effects of the osteoblast lineage versus adipocyte lineage and other stromal cells on MM cells.

\section{Acknowledgments}

We thank Dr. Ralph D. Sanderson (University of Alabama at Birmingham, Birmingham, AL) for CAG MM cells, Dr. Fenghuang Zhan (University of Iowa, Iowa City, IA) for 5TGM1-luc MM cells, Dr. Timothy Garvey (University of Alabama at Birmingham) for 3T3-L1 preadipocytes, and Dr. Deniz Peker (University of Alabama at Birmingham) for MM patient samples. We also thank Dr. Kurt R. Zinn and the UAB Animal Imaging Core for assistance with mouse bioluminescence imaging and the UAB Histomorphometry and Molecular Analysis Core for tissue processing.

\section{References}

1. de Haart SJ, van de Donk NW, Minnema MC, Huang JH, AartsRiemens T, Bovenschen N, Yuan H, Groen RW, McMillin DW, Jakubikova J, Lokhorst HM, Martens AC, Mitsiades CS, Mutis T: Accessory cells of the microenvironment protect multiple myeloma from T-cell cytotoxicity through cell adhesion-mediated immune resistance. Clin Cancer Res 2013, 19:5591-5601

2. Dankbar B, Padro T, Leo R, Feldmann B, Kropff M, Mesters RM, Serve H, Berdel WE, Kienast J: Vascular endothelial growth factor and interleukin-6 in paracrine tumor-stromal cell interactions in multiple myeloma. Blood 2000, 95:2630-2636

3. Nefedova Y, Landowski TH, Dalton WS: Bone marrow stromal derived soluble factors and direct cell contact contribute to de novo drug resistance of myeloma cells by distinct mechanisms. Leukemia 2003, 17:1175-1182

4. Parmo-Cabanas M, Bartolome RA, Wright N, Hidalgo A, Drager AM, Teixido J: Integrin alpha4betal involvement in stromal cell-derived factor-1alpha-promoted myeloma cell transendothelial migration and adhesion: role of cAMP and the actin cytoskeleton in adhesion. Exp Cell Res 2004, 294:571-580

5. Richardson PG, Schlossman RL, Weller E, Hideshima T, Mitsiades C, Davies F, LeBlanc R, Catley LP, Doss D, Kelly K, McKenney M, Mechlowicz J, Freeman A, Deocampo R, Rich R, Ryoo JJ, Chauhan D, Balinski K, Zeldis J, Anderson KC: Immunomodulatory drug CC-5013 overcomes drug resistance and is well tolerated in patients with relapsed multiple myeloma. Blood 2002, 100:3063-3067

6. Hideshima T, Chauhan D, Hayashi T, Akiyama M, Mitsiades N, Mitsiades C, Podar K, Munshi NC, Richardson PG, Anderson KC: Proteasome inhibitor PS-341 abrogates IL-6 triggered signaling cascades via caspase-dependent downregulation of gp130 in multiple myeloma. Oncogene 2003, 22:8386-8393

7. Yang Y, Ren Y, Ramani VC, Nan L, Suva LJ, Sanderson RD: Heparanase enhances local and systemic osteolysis in multiple myeloma by upregulating the expression and secretion of RANKL. Cancer Res 2010, 70:8329-8338

8. Ruan J, Trotter TN, Nan L, Luo R, Javed A, Sanderson RD, Suva LJ, Yang Y: Heparanase inhibits osteoblastogenesis and shifts bone marrow progenitor cell fate in myeloma bone disease. Bone 2013, 57:10-17

9. Sceneay J, Smyth MJ, Moller A: The pre-metastatic niche: finding common ground. Cancer Metastasis Rev 2013, 32:449-464

10. Kawano Y, Moschetta M, Manier S, Glavey S, Görgün GT, Roccaro AM, Anderson KC, Ghobrial IM: Targeting the bone marrow microenvironment in multiple myeloma. Immunol Rev 2015, 263: $160-172$ 
11. Toscani D, Bolzoni M, Accardi F, Aversa F, Giuliani N: The osteoblastic niche in the context of multiple myeloma. Ann N Y Acad Sci 2015, 1335:45-62

12. Fischer-Posovszky P, Wabitsch M, Hochberg Z: Endocrinology of adipose tissue - an update. Horm Metab Res 2007, 39:314-321

13. Asosingh K, Radl J, Van Riet I, Van Camp B, Vanderkerken K: The 5TMM series: a useful in vivo mouse model of human multiple myeloma. Hematol J 2000, 1:351-356

14. Dallas SL, Garrett IR, Oyajobi BO, Dallas MR, Boyce BF, Bauss F, Radl J, Mundy GR: Ibandronate reduces osteolytic lesions but not tumor burden in a murine model of myeloma bone disease. Blood 1999, 93:1697-1706

15. Radl J, De Glopper E, Schuit HR, Zurcher C: Idiopathic paraproteinemia, II: transplantation of the paraprotein-producing clone from old to young C57BL/KaLwRij mice. J Immunol 1979, 122:609-613

16. Trotter TN, Li M, Pan Q, Peker D, Rowan PD, Li J, Zhan F, Suva LJ, Javed A, Yang Y: Myeloma cell-derived Runx2 promotes myeloma progression in bone. Blood 2015, 125:3598-3608

17. Purushothaman A, Chen L, Yang Y, Sanderson RD: Heparanase stimulation of protease expression implicates it as a master regulator of the aggressive tumor phenotype in myeloma. J Biol Chem 2008, 283: 32628-32636

18. Wang Y, Kim KA, Kim JH, Sul HS: Pref-1, a preadipocyte secreted factor that inhibits adipogenesis. J Nutr 2006, 136:2953-2956

19. Parlee SD, Lentz SI, Mori H, MacDougald OA: Quantifying size and number of adipocytes in adipose tissue. Methods Enzymol 2014, 537: $93-122$

20. Takeshita S, Fumoto T, Naoe Y, Ikeda K: Age-related marrow adipogenesis is linked to increased expression of RANKL. J Biol Chem 2014, 289:16699-16710

21. Gao D, Trayhurn P, Bing C: 1,25-Dihydroxyvitamin D3 inhibits the cytokine-induced secretion of MCP-1 and reduces monocyte recruitment by human preadipocytes. Int J Obes (2005) 2013, 37:357-365

22. Vande Broek I, Asosingh K, Allegaert V, Leleu X, Facon T, Vanderkerken K, Van Camp B, Van Riet I: Bone marrow endothelial cells increase the invasiveness of human multiple myeloma cells through upregulation of MMP-9: evidence for a role of hepatocyte growth factor. Leukemia 2004, 18:976-982

23. Tanaka Y, Abe M, Hiasa M, Oda A, Amou H, Nakano A, Takeuchi K, Kitazoe K, Kido S, Inoue D, Moriyama K, Hashimoto T, Ozaki S, Matsumoto T: Myeloma cell-osteoclast interaction enhances angiogenesis together with bone resorption: a role for vascular endothelial cell growth factor and osteopontin. Clin Cancer Res 2007, 13:816-823

24. Vande Broek I, Asosingh K, Vanderkerken K, Straetmans N, Van Camp B, Van Riet I: Chemokine receptor CCR2 is expressed by human multiple myeloma cells and mediates migration to bone marrow stromal cell-produced monocyte chemotactic proteins MCP-1, -2 and -3. Br J Cancer 2003, 88:855-862

25. Roccaro Aldo M, Sacco A, Purschke Werner G, Moschetta M, Buchner K, Maasch C, Zboralski D, Zöllner S, Vonhoff S, Mishima Y, Maiso P, Reagan Michaela R, Lonardi S, Ungari M, Facchetti F, Eulberg D, Kruschinski A, Vater A, Rossi G, Klussmann S, Ghobrial Irene M: SDF-1 inhibition targets the bone marrow niche for cancer therapy. Cell Rep 2014, 9:118-128

26. Pellegrino A, Ria R, Di Pietro G, Cirulli T, Surico G, Pennisi A, Morabito F, Ribatti D, Vacca A: Bone marrow endothelial cells in multiple myeloma secrete CXC-chemokines that mediate interactions with plasma cells. Br J Haematol 2005, 129:248-256

27. Wang Y, Sul HS: Ectodomain shedding of preadipocyte factor 1 (Pref-1) by tumor necrosis factor alpha converting enzyme (TACE) and inhibition of adipocyte differentiation. Mol Cell Biol 2006, 26:5421-5435

28. Kolf CM, Cho E, Tuan RS: Mesenchymal stromal cells. Biology of adult mesenchymal stem cells: regulation of niche, self-renewal and differentiation. Arthritis Res Ther 2007, 9:204

29. Moerman EJ, Teng K, Lipschitz DA, Lecka-Czernik B: Aging activates adipogenic and suppresses osteogenic programs in mesenchymal marrow stroma/stem cells: the role of PPAR-gamma2 transcription factor and TGF-beta/BMP signaling pathways. Aging Cell 2004, 3:379-389

30. Justesen J, Stenderup K, Ebbesen EN, Mosekilde L, Steiniche T, Kassem M: Adipocyte tissue volume in bone marrow is increased with aging and in patients with osteoporosis. Biogerontology 2001, 2:165-171

31. Giuliani N, Colla S, Morandi F, Lazzaretti M, Sala R, Bonomini S, Grano M, Colucci S, Svaldi M, Rizzoli V: Myeloma cells block RUNX2/CBFA1 activity in human bone marrow osteoblast progenitors and inhibit osteoblast formation and differentiation. Blood 2005, 106:2472-2483

32. Skurk T, Alberti-Huber C, Herder C, Hauner H: Relationship between adipocyte size and adipokine expression and secretion. J Clin Endocrinol Metab 2007, 92:1023-1033

33. Jacobsson B, Smith U: Effect of cell size on lipolysis and antilipolytic action of insulin in human fat cells. J Lipid Res 1972, 13:651-656

34. Gilbert CA, Slingerland JM: Cytokines, obesity, and cancer: new insights on mechanisms linking obesity to cancer risk and progression. Annu Rev Med 2013, 64:45-57

35. Hardaway AL, Herroon MK, Rajagurubandara E, Podgorski I: Bone marrow fat: linking adipocyte-induced inflammation with skeletal metastases. Cancer Metastasis Rev 2014, 33:527-543

36. Vande Broek I, Vanderkerken K, Van Camp B, Van Riet I: Extravasation and homing mechanisms in multiple myeloma. Clin Exp Metastasis 2008, 25:325-334

37. Tirado-Velez JM, Joumady I, Saez-Benito A, Cozar-Castellano I, Perdomo G: Inhibition of fatty acid metabolism reduces human myeloma cells proliferation. PLoS One 2012, 7:e46484

38. Fujiwara S, Wada N, Kawano Y, Okuno Y, Kikukawa Y, Endo S, Nishimura N, Ueno N, Mitsuya H, Hata H: Lactate, a putative survival factor for myeloma cells, is incorporated by myeloma cells through monocarboxylate transporters 1. Exp Hematol Oncol 2015, 4:12

39. Nieman KM, Kenny HA, Penicka CV, Ladanyi A, Buell-Gutbrod R, Zillhardt MR, Romero IL, Carey MS, Mills GB, Hotamisligil GS, Yamada SD, Peter ME, Gwin K, Lengyel E: Adipocytes promote ovarian cancer metastasis and provide energy for rapid tumor growth. Nat Med 2011, 17:1498-1503

40. Spranger S, Bao R, Gajewski TF: Melanoma-intrinsic beta-catenin signalling prevents anti-tumour immunity. Nature 2015, 523:231-235

41. Tagde A, Rajabi H, Bouillez A, Alam M, Gali R, Bailey S, Tai YT, Hideshima T, Anderson K, Avigan D, Kufe D: MUC1-C drives MYC in multiple myeloma. Blood 2016, 127:2587-2597

42. Muller T, Bain G, Wang X, Papkoff J: Regulation of epithelial cell migration and tumor formation by beta-catenin signaling. Exp Cell Res 2002, 280:119-133

43. Lentzsch S, Chatterjee M, Gries M, Bommert K, Gollasch $H$, Dorken B, Bargou RC: PI3-K/AKT/FKHR and MAPK signaling cascades are redundantly stimulated by a variety of cytokines and contribute independently to proliferation and survival of multiple myeloma cells. Leukemia 2004, 18:1883-1890

44. Ge NL, Rudikoff S: Insulin-like growth factor I is a dual effector of multiple myeloma cell growth. Blood 2000, 96:2856-2861

45. Fulda S: Targeting apoptosis for anticancer therapy. Semin Cancer Biol 2015, 31:84-88

46. Yaccoby S, Wezeman MJ, Zangari M, Walker R, Cottler-Fox M, Gaddy D, Ling W, Saha R, Barlogie B, Tricot G, Epstein J: Inhibitory effects of osteoblasts and increased bone formation on myeloma in novel culture systems and a myelomatous mouse model. Haematologica 2006, 91:192-199

47. Takeuchi K, Abe M, Hiasa M, Oda A, Amou H, Kido S, Harada T, Tanaka O, Miki H, Nakamura S, Nakano A, Kagawa K, Yata K, Ozaki S, Matsumoto T: Tgf-Beta inhibition restores terminal osteoblast differentiation to suppress myeloma growth. PLoS One 2010, 5:e9870

48. Yaccoby S, Ling W, Zhan F, Walker R, Barlogie B, Shaughnessy JD Jr: Antibody-based inhibition of DKK1 suppresses tumor-induced bone resorption and multiple myeloma growth in vivo. Blood 2007, 109:2106-2111 